\title{
Mono-Higgs detection of dark matter at the LHC
}

\author{
Asher Berlin, Tongyan Lin and Lian-Tao Wang \\ Enrico Fermi Institute and Kavli Institute for Cosmological Physics, \\ The University of Chicago, Chicago, Illinois, 60637-1433, U.S.A. \\ E-mail: berlin@uchicago.edu, tongyan@kicp.uchicago.edu, \\ liantaow@uchicago.edu
}

ABSTRACT: Motivated by the recent discovery of the Higgs boson, we investigate the possibility that a missing energy plus Higgs final state is the dominant signal channel for dark matter at the LHC. We consider examples of higher-dimension operators where a Higgs and dark matter pair are produced through an off-shell $Z$ or $\gamma$, finding potential sensitivity at the LHC to cutoff scales of around a few hundred GeV. We generalize this production mechanism to a simplified model by introducing a $Z^{\prime}$ as well as a second Higgs doublet, where the pseudoscalar couples to dark matter. Resonant production of the $Z^{\prime}$ which decays to a Higgs plus invisible particles gives rise to a potential mono-Higgs signal. This may be observable at the $14 \mathrm{TeV}$ LHC at low $\tan \beta$ and when the $Z^{\prime}$ mass is roughly in the range $600 \mathrm{GeV}$ to $1.3 \mathrm{TeV}$.

Keywords: Phenomenological Models, Hadronic Colliders

ARXIV EPRINT: 1402.7074 


\section{Contents}

1 Introduction 1

2 Higgs + MET at the LHC 3

2.1 Two b-jet channel 3

2.2 Diphoton channel 4

3 Effective field theory 5

$\begin{array}{lll}3.1 & \text { Unitarity } & 7\end{array}$

4 Dark matter via a $Z^{\prime}$ and heavy Higgs $\quad 8$

$\begin{array}{lll}4.1 Z^{\prime} \text { constraints } & 10\end{array}$

4.2 Mono-Higgs signal 11

$\begin{array}{lll}4.3 & \text { Dark matter coupling to Higgs sector } & 14\end{array}$

5 Summary and conclusions $\quad 15$

\section{Introduction}

Dark matter (DM) contributes a large component of the mass-energy of the universe. The leading hypothesis is that most of the dark matter is in the form of stable, electrically neutral, massive particles, which interact at least gravitationally with baryons. If such a particle interacts non-gravitationally with standard model (SM) particles as well, for instance via the weak force, detecting it through high-energy collisions at particle accelerators is one of the most promising avenues towards identifying the specific nature of its detailed interactions. For instance, if DM production is kinematically accessible at the Large Hadron Collider (LHC), then missing energy signatures that deviate from SM predictions would provide compelling evidence of new stable, electrically neutral particles, and thus strong candidates for cosmological DM.

Various approaches to describing particle DM interactions have been explored in order to understand possible detection signatures at the LHC. The most detailed set of attempts include complete quantum field theories incorporating many new particles into the SM, for example supersymmetric dark matter [1]. Such top-down, or UV-complete, theories often have large sets of a priori undetermined additional parameters. Therefore, making confident phenomenological predictions can become very burdensome.

On the opposite end of the spectrum, one can assume that aside from the dark matter, any new heavy fields of the UV-complete theory can be integrated out and that the relevant physics can be described by an effective field theory (EFT). The effective field theorist thus proceeds by writing down a tower of non-renormalizable contact operators governing the 

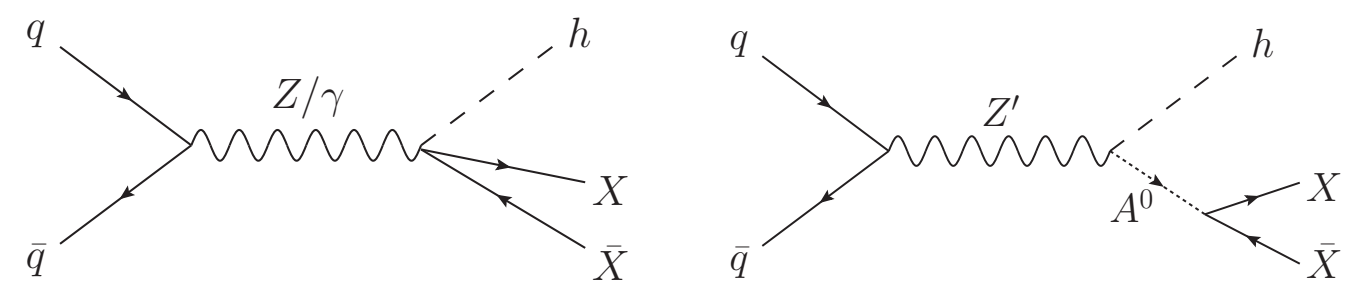

Figure 1. Production mechanisms for dark matter plus Higgs through (left) a contact operator coupling dark matter to $Z h$ or $\gamma h$, or (right) a new $Z^{\prime}$ coupled to a two Higgs doublet model, where the new pseudoscalar $A^{0}$ decays primarily to the dark matter.

DM interactions with SM particles. The underlying UV theory determines the coefficients of these operators, which in turn can be constrained in a model-independent way from experimental results and also be related in a simple way to relic density or direct detection predictions [2]. In addition to the relative simplicity of constraining individual operators, this approach has a particular appeal at a time when no other signs of new physics have yet been discovered at the LHC.

Recent studies taking advantage of the EFT technique have considered collider signals such as monophoton [3], monojet [4-7], mono- $Z / W[8,9]$ and mono- $b$ events [10], during which one (or more) particle of the SM is produced and detected in the collider, recoiling against some missing transverse energy (MET or $\not p_{T}$ ) associated with the DM. This work has been generalized to a set of so-called "simplified models" where the DM couples to the SM through renormalizable interactions, for example through a new mediator that can be produced on-shell [11-15].

In light of the recent Higgs discovery at the LHC $[16,17]$, we can expand our search in yet another avenue. In this paper we investigate the possible production of a Higgs along with DM, which is accordingly dubbed a mono-Higgs process. The observed final states are MET plus the Higgs decay products, with an invariant mass constrained to be relatively close to the true mass $m_{h} \approx 125 \mathrm{GeV}$.

The focus of our article is to explore those possibilities where mono-Higgs could be the primary production mechanism for DM at the LHC. We will consider examples of both contact operators and simplified models. We begin in section 2 with a discussion of LHC searches for Higgs plus MET final states, concentrating here on the $b \bar{b}$ and diphoton decay channels for the Higgs. In section 3, we present examples of higher-dimension operators coupling DM to Higgs doublets and electroweak gauge bosons. We derive constraints on the coefficients of these operators both with and without implementing a unitarity condition on the potential signal events. Motivated by the processes in the EFT description, in section 4 we introduce a simplified model with a $Z^{\prime}$ gauge boson and two Higgs doublets, where the dark matter is coupled to the heavy pseudoscalar Higgs. We demonstrate that the $14 \mathrm{TeV}$ LHC can probe the parameter space of this model at low $\tan \beta$. We conclude in section 5.

We also note that the mono-Higgs signal has recently been discussed in refs. [18, 19]. Ref. [18] considered contact operators coupling dark matter to SM Higgs doublets and possibly other SM states (the operators are different from the ones in this paper); however 
they found that for most of the operators the bounds on the cutoff scale are quite low, less than $50 \mathrm{GeV}$, which is well beyond the regime of validity for assuming a contact operator.

Ref. [19] considered a somewhat different set of operators as well as simplified models. For the "Higgs-portal"-type operators (e.g., [20-22]), they find LHC limits to be much weaker than exclusion limits on Higgs invisible decay for DM masses below $m_{h} / 2$, while direct detection is very constraining at higher masses. Ref. [19] also considered simplified models with an additional $Z^{\prime}$, where the Higgs is produced through Higgs-strahlung of the $Z^{\prime}$. For the case of $Z-Z^{\prime}$ mass mixing, they found mono-Higgs is only able to probe large mixing angles $(\sin \theta>0.1)$, in apparent conflict with precision electroweak data. In contrast, for our scenario the $Z^{\prime}$ is produced resonantly and decays, and we have imposed the precision electroweak constraint from fits of the $\rho_{0}$ parameter.

\section{Higgs + MET at the LHC}

We consider two possible Higgs decay channels, $b \bar{b}$ and $\gamma \gamma$, as promising for observing Higgs plus MET. The $b \bar{b}$ channel has the largest branching ratio for a Higgs of mass $m_{h}=125 \mathrm{GeV}, \operatorname{Br}(h \rightarrow b \bar{b}) \approx 0.577$ [23], and gives the best statistics for the signal, while the diphoton branching ratio is only $\operatorname{Br}(h \rightarrow \gamma \gamma) \approx 2.28 \times 10^{-3}$, but is potentially a very clean channel. These channels as well as multi-lepton final states from $h \rightarrow Z Z^{*}$ were also studied in [19].

The dominant irreducible SM background for Higgs plus MET is $Z h$ production with $Z$ decaying to neutrinos. Depending on the decay channel, other SM backgrounds can also be comparable or larger. Here we rely on the ATLAS report [24] to derive bounds from LHC Run 1. For $14 \mathrm{TeV}$ projections, we estimate backgrounds rates from our own Monte Carlo event simulations and also use some results from [19].

Our dark matter models have been implemented with FeynRules 2.0 [25], and our event generation makes use of the MadGraph [26], PYTHIA [27], and Delphes [28] pipeline from parton-level to detector-level simulation.

\subsection{Two b-jet channel}

A search for $h \rightarrow b \bar{b}$ decay in association with a $Z / W$ boson has been performed using the data of Run 1 of the LHC; the observed signal strength is compatible with that of the SM Higgs boson [24, 29]. In particular, the ATLAS collaboration presents an analysis for the $Z(\nu \bar{\nu}) h$ channel in several MET bins, with the full integrated luminosity of $4.7 / \mathrm{fb}$ at $7 \mathrm{TeV}$ and $20.3 / \mathrm{fb}$ at $\sqrt{s}=8 \mathrm{TeV}$ [24]. We use these results to derive constraints on mono-Higgs for the models in this paper.

Event selection is governed by demanding two leading $b$-tagged jets, with $p_{T}>20 \mathrm{GeV}$ and $|\eta|<2.5$, with the highest $p_{T} b$-tagged jet having $p_{T}>45 \mathrm{GeV}$. Multijet backgrounds are reduced by requiring $\mathbb{E}_{T}>120 \mathrm{GeV}$ and constraints on the azimuthal angle between the missing transverse momentum and jets: $\Delta \phi\left(\mathbb{E}_{T}, b \bar{b}\right)>2.8, \min \left[\Delta \phi\left(\mathbb{E}_{T}, j\right)\right]>1.5$. A lepton veto is imposed, and the $b \bar{b}$ system invariant mass must reconstruct to near the Higgs mass, $90 \mathrm{GeV}<m_{b b}<150 \mathrm{GeV}$. Finally, $t \bar{t}$ is suppressed by vetoing events that have any additional jets with $p_{T}>30 \mathrm{GeV}$. 


\begin{tabular}{|c|c|c|}
\hline & LHC Run 1 & $14 \mathrm{TeV}$ \\
\hline$t \bar{t}$ & 200 & $1006 \pm 335$ \\
$Z b \bar{b}$ & 336 & $682 \pm 26$ \\
$V h$ & 23 & $142 \pm 5$ \\
SM total & $727 \pm 11$ & $1830 \pm 336$ \\
\hline Dim-8, fermion DM & $329 \pm 10$ & $23150 \pm 880$ \\
$M_{Z^{\prime}}=1 \mathrm{TeV}, \tan \beta=1$ & $43 \pm 1$ & $1836 \pm 36$ \\
\hline
\end{tabular}

Table 1. Background and signal events for $h \rightarrow b \bar{b}$ decay, for the cuts described in the text. The background numbers for LHC Run I are taken from ref. [24] for MET $>120 \mathrm{GeV}$. For our background estimate at a $14 \mathrm{TeV} \mathrm{LHC}$, we include only the processes listed here; uncertainties from MC statistics are shown and we include an additional $25 \%$ systematic uncertainty in deriving constraints. For the signal from a dimension-8 operator with fermion DM, eq. (3.7), we take fiducial values of $\Lambda=200 \mathrm{GeV}$ and $m_{X}=1 \mathrm{GeV}$. For the $Z^{\prime}$ case, the coupling is the upper limit allowed by the $\rho_{0}$ constraint, shown in figure 4 .

Estimates of SM processes, including $Z h$, are compared to observed data events in three MET bins. The most important backgrounds are $Z+b \bar{b}$ and $t \bar{t}$. Making use of these published SM process estimates, we compare our signal to the data with cuts of $\mathbb{E}_{T}>120 \mathrm{GeV}, \mathbb{E}_{T}>160 \mathrm{GeV}$, and $\mathbb{E}_{T}>200 \mathrm{GeV}$, and derive $95 \% \mathrm{CL}$ upper limits on the number of possible mono-Higgs signal events. We have also validated our event simulation against these background estimates.

For $14 \mathrm{TeV}$ projections, we modify the $8 \mathrm{TeV}$ ATLAS cuts slightly, loosing the jet veto such that up to one additional jet with $p_{T}>30 \mathrm{GeV}$ is allowed, and take a cut of $\mathbb{E}_{T}>250 \mathrm{GeV}$. The total integrated luminosity is $300 / \mathrm{fb}$. Our estimates for background rates are shown in table 1 . We find the $b \bar{b}$ channel performs better compared to the results in ref. [19]; this appears to be due primarily to our choice of $R=0.4$ jet clustering radius instead of $R=0.7$, since with a larger radius the two $b$-jets from the Higgs decay are more often clustered together in the boosted Higgs regime.

\subsection{Diphoton channel}

The diphoton channel requires two hard photons reconstructing to the Higgs mass, large missing energy, and a veto on leptons. The dominant SM backgrounds are $Z \gamma \gamma$ and $h Z / h W$. Because the Higgs branching ratio to photons is so small, we find that this channel is not constraining if the $8 \mathrm{TeV}$ run is considered, since there are simply not enough signal events. However, the statistics are far improved at $14 \mathrm{TeV}$. We use results for background estimates from [19], where they found that this channel can demonstrate improved sensitivity over $b \bar{b}$ (which suffers from a larger $t \bar{t}$ background). The cuts applied require $m_{\gamma \gamma} \in[110,130] \mathrm{GeV}$ and $\mathbb{E}_{T}>100,250 \mathrm{GeV}$ at $8,14 \mathrm{TeV}$ respectively. 

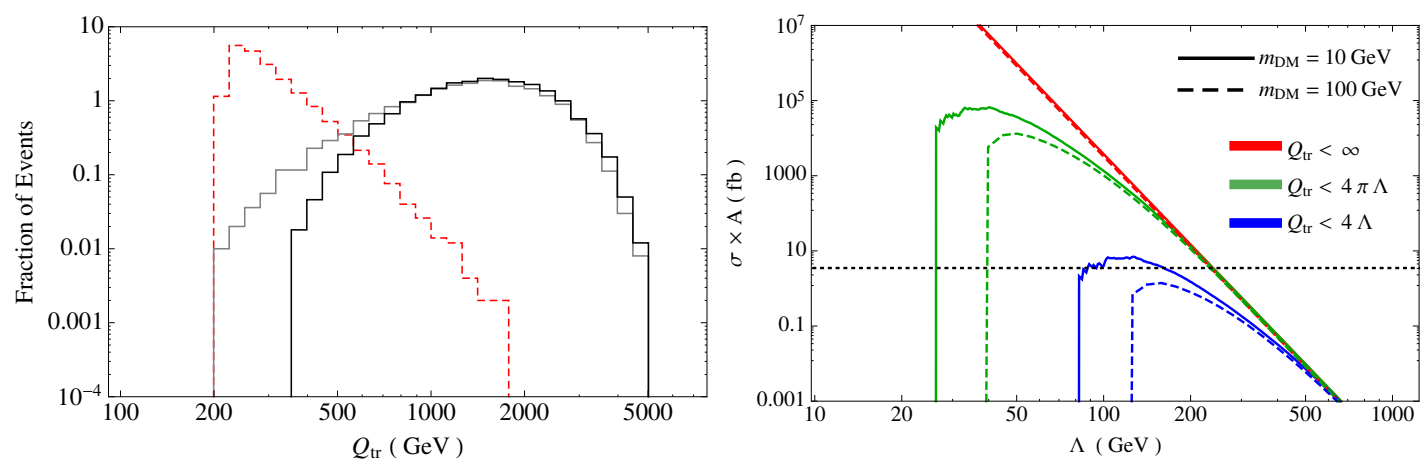

Figure 2. Left: distributions (at $\sqrt{s}=8 \mathrm{TeV}$ ) for the momentum transfer $Q_{\mathrm{tr}}$ for $10 \mathrm{GeV}$ (solid gray line) and $100 \mathrm{GeV}$ (solid black line) DM, from a mono-Higgs DM signal corresponding to the operator of eq. (3.7) with fermion DM. The irreducible SM background from $Z h$ production (red dashed line) is also shown. Right: for the same operator, the rate for mono-Higgs at $8 \mathrm{TeV}$ with a cut of $120 \mathrm{GeV}$ missing transverse energy. The total cross section is scaled by the fraction of events satisfying various "unitarity" conditions on $Q_{\mathrm{tr}}$. The horizontal line indicates the approximate cross section that would be ruled out at 95\% CL using data from Run 1 of the LHC; regions of $\Lambda$ with cross sections above this line are excluded and correspond to the shaded regions in figure 3.

\section{Effective field theory}

Contact operators coupling dark matter to a Higgs doublet can potentially give rise to a mono-Higgs signal. If the dark matter is a gauge singlet, then gauge invariance implies the operator must also include other electroweak doublets. We focus on operators that give rise to a coupling of dark matter to both $h$ and $Z / \gamma$, allowing the production of dark matter through the process shown in figure 1. If the dark matter couples to two Higgs bosons, the production rate is correspondingly lower.

For the process above, we also note that a mono- $Z$ signal is possible by reversing the roles of the $h$ and the $Z$; this rate is automatically lower by several orders of magnitude since it requires the initial production of an $s$-channel Higgs. For all the operators considered here, the limits from mono- $Z$ are weaker compared to mono-Higgs.

These kinds of operators have been studied in refs. [19, 30, 31], as well as mono-Higgs from Higgs-portal type operators in [18]. The lowest dimension SM operator that can give a $Z h$ interaction with dark matter is

$$
i\left(H^{\dagger} D_{\mu} H-\text { h.c. }\right) \rightarrow-2 m_{Z} h Z_{\mu}-\langle v\rangle m_{Z} Z_{\mu},
$$

after electroweak symmetry breaking. This operator could be combined with singlets formed of the dark matter: $i\left(\phi^{\dagger} \partial^{\mu} \phi-\right.$ h.c. $)$ for scalar DM, and $\bar{X} \gamma^{\mu} X$ or $\bar{X} \gamma^{\mu} \gamma^{5} X$ for fermion DM. Because of the induced direct $Z$ coupling to dark matter, direct detection is very constraining for $m_{\mathrm{DM}}>10 \mathrm{GeV}$, while the invisible $Z$ width is very constraining for $m_{\mathrm{DM}}<m_{Z} / 2$. Despite this, in the case of scalar DM ref. [19] found that a mono-Higgs search at $14 \mathrm{TeV}$ could be much more sensitive than the invisible $Z$ width. ${ }^{1}$ We therefore do not consider this operator further.

\footnotetext{
${ }^{1}$ The constraints on the suppression scale $\Lambda$ are again of order a few hundred GeV up to a TeV for the LHC and therefore has the same problem with unitarity that we discuss below.
} 

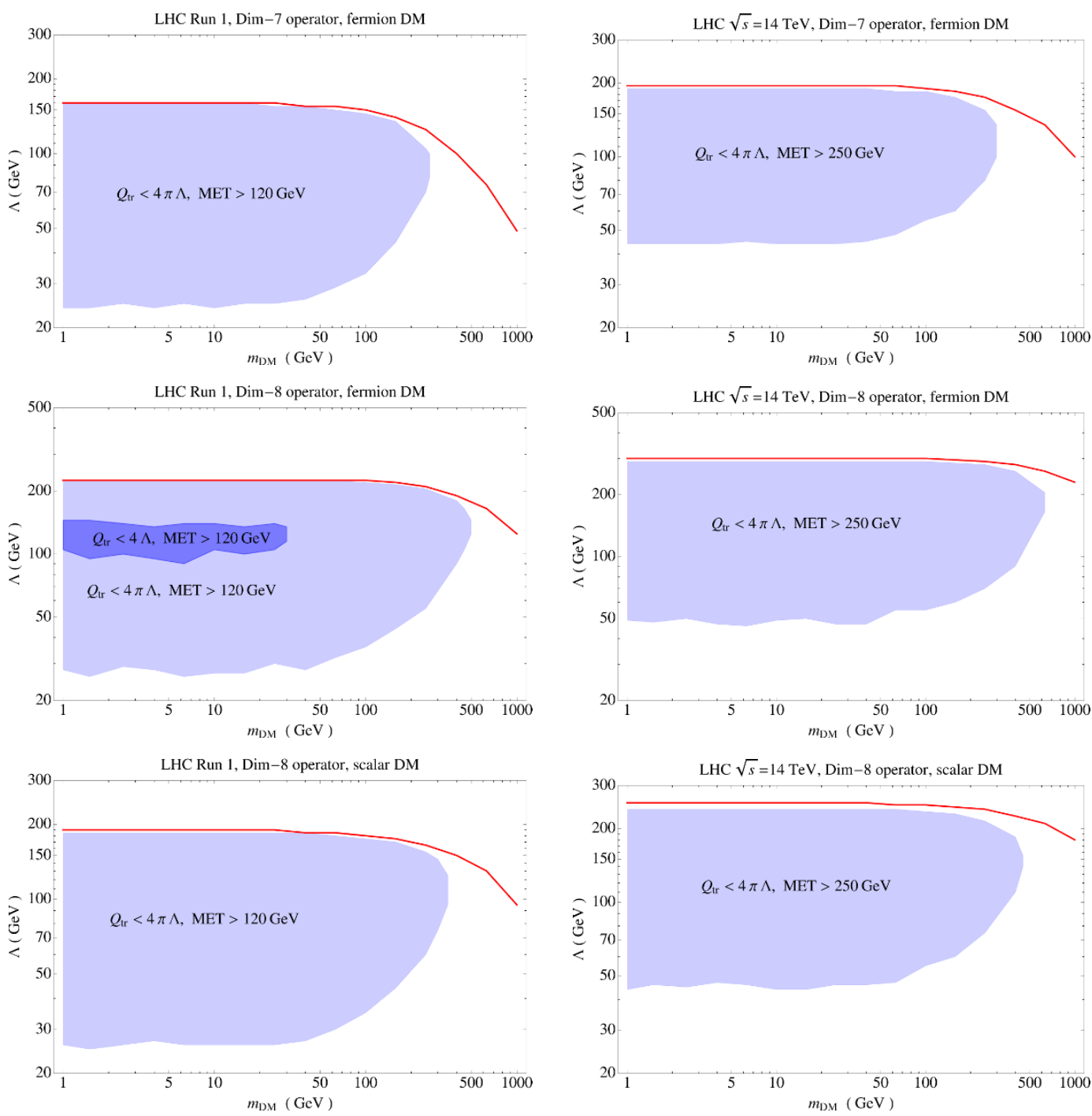

Figure 3. $95 \%$ CL constraints from mono-Higgs on the suppression scale $\Lambda$ as a function of DM mass, for operators discussed in section 3. The dimension-7 operator is $\frac{i}{\Lambda^{3}} \bar{X} \gamma^{\mu \nu} X\left[\left(D_{\mu} H\right)^{\dagger} D_{\nu} H\right.$ - h.c. $]$, the dimension-8 operator coupling to fermion DM is $\frac{1}{\Lambda^{4}} \bar{X} \gamma^{\mu} X$ (W $W_{\nu \mu}^{a} H^{\dagger} t^{a} D^{\nu} H+$ h.c.), and the dimension-8 operator coupling to scalar DM is $\frac{1}{\Lambda^{4}} \frac{1}{2}\left(\phi^{\dagger} \partial^{\mu} \phi+\right.$ h.c. $)\left(B_{\nu \mu} H^{\dagger} D^{\nu} H+\right.$ h.c. $)$. The solid lines are the lower bounds for the naive EFT result $\left(Q_{\mathrm{tr}}<\infty\right)$. The shaded regions are the excluded regions imposing the conditions on the momentum transfer $Q_{\mathrm{tr}}<4 \pi \times \Lambda$ or $Q_{\mathrm{tr}}<4 \Lambda$ to address the apparent violation of unitarity. The left column shows LHC Run 1 limits, derived for the $b \bar{b}$ channel with a MET cut of $120 \mathrm{GeV}$, while right column shows $14 \mathrm{TeV}$ limits assuming the diphoton channel and a MET cut of $250 \mathrm{GeV}$.

At dimension-4 in the SM factor there is the operator

$$
i\left[\left(D_{\mu} H\right)^{\dagger} D_{\nu} H-\text { h.c. }\right] \rightarrow m_{Z}\left(Z_{\mu} \partial_{\nu} h-Z_{\nu} \partial_{\mu} h\right),
$$

concentrating on the part giving an $h Z$ interaction. Including a DM factor, we consider

$$
\frac{1}{\Lambda^{3}} \bar{X} \gamma^{\mu \nu} X \times i\left[\left(D_{\mu} H\right)^{\dagger} D_{\nu} H-\text { h.c. }\right] \text {, }
$$

neglecting the similar possibility with $\bar{X} \gamma^{5} \gamma^{\mu \nu} X$. 
Finally there are dimension-5 SM operators [30]

$$
\begin{aligned}
& \left(B_{\nu \mu} Y_{H} H^{\dagger} D^{\nu} H+\text { h.c. }\right) \\
& \quad \rightarrow \frac{\langle v\rangle}{2}\left(\cos \theta_{w} F_{\nu \mu} \partial^{\nu} h-\sin \theta_{w} Z_{\nu \mu} \partial^{\nu} h\right) \\
& \left(W_{\nu \mu}^{a} H^{\dagger} t^{a} D^{\nu} H+\text { h.c. }\right) \\
& \quad \rightarrow-\frac{\langle v\rangle}{2}\left(\sin \theta_{w} F_{\nu \mu} \partial^{\nu} h+\cos \theta_{w} Z_{\nu \mu} \partial^{\nu} h\right)
\end{aligned}
$$

where $B_{\nu \mu}, W_{\nu \mu}^{a}$ are the field strengths for $\mathrm{U}(1)_{Y}$ and $\mathrm{SU}(2)_{L}$, and $Z_{\nu \mu}, F_{\nu \mu}$ are the field strengths for $Z$ and $\gamma$, respectively. Dimension- 8 operators are formed by including a DM factor of either $\bar{X} \gamma^{\mu} X$ or $\bar{X} \gamma^{\mu 5} X$ for fermion DM, and either $i\left(\phi^{\dagger} \partial^{\mu} \phi-h . c.\right)$ or $\left(\phi^{\dagger} \partial^{\mu} \phi+\right.$ h.c. $)$ for scalar DM. Combined with the possibility of exchanging $B_{\nu \mu}, W_{\nu \mu}^{a}$ for $\tilde{B}_{\nu \mu}, \tilde{W}_{\nu \mu}^{a}$, a large number of operators are possible. We therefore restrict our attention to two representative examples with scalar DM $(\phi)$ or a Dirac fermion $(X)$ :

$$
\begin{aligned}
& \frac{1}{\Lambda^{4}} \frac{1}{2}\left(\phi^{\dagger} \partial^{\mu} \phi+\text { h.c. }\right)\left(B_{\nu \mu} H^{\dagger} D^{\nu} H+\text { h.c. }\right) \\
& \frac{1}{\Lambda^{4}} \bar{X} \gamma^{\mu} X\left(W_{\nu \mu}^{a} H^{\dagger} t^{a} D^{\nu} H+\text { h.c. }\right),
\end{aligned}
$$

refs. [30, 31] discuss the complete list of possible operators, as well as further details on the relic density and gamma-ray signals of dark matter annihilation.

For the operators in eqs. (3.3), and (3.6)-(3.7) we derive constraints on $\Lambda$ as a function of DM mass from a mono-Higgs search. For LHC Run 1 data, we consider the $b \bar{b}$ channel with the weakest cut on the missing energy $\mathbb{E}_{T}>120 \mathrm{GeV}$. Higher $\mathbb{E}_{T}$ values will necessarily require larger momentum transfer and thus lead to even larger error in the validity of the EFT, as discussed further in the following section. For $14 \mathrm{TeV}$, we obtain constraints using the diphoton channel, where we find the best results.

The LHC Run 1 lower bounds on $\Lambda$ are comparable and on the order of $200 \mathrm{GeV}$ for all three cases, increasing up to $300 \mathrm{GeV}$ for $14 \mathrm{TeV}$ projections. The related operator $\frac{1}{\Lambda^{4}} \bar{X} \gamma^{\mu} X\left(B_{\mu \nu} H^{\dagger} D^{\nu} H+\right.$ h.c. $)$ was also studied in ref. [19], where they obtained very similar bounds.

Even though one would expect the constraints on the dimension-7 operator to be stronger than for the dimension- 8 ones, they are in fact slightly weaker. This is because most of the mono-Higgs signal is coming from the high momentum transfer $\left(Q_{\mathrm{tr}}\right)$ region, as can also be seen in figure 2, and the dimension-7 operator has a softer $Q_{\operatorname{tr}}$ dependence. This result is clearly related to the issue of validity of the EFT, as we discuss further below.

\subsection{Unitarity}

A frequent concern in this EFT approach is that, taking LHC constraints at face value, the values of $\Lambda$ that can be probed correspond to energy scales accessible at the LHC. This implies a violation of perturbative unitarity at high momentum transfer, or equivalently that the EFT is no longer a valid description for LHC processes.

Figure 2 shows the distribution for the momentum transfer $Q_{\mathrm{tr}}$ for the operator of eq. (3.7). Compared to the naive constraint of $\Lambda \gtrsim 225 \mathrm{GeV}$ derived for the operator, 
it is clear that the EFT description is on shaky footing. For an $s$-channel mediator, the condition $Q_{\operatorname{tr}} \lesssim 4 \pi \Lambda$ is required for an expansion in the mediator mass for a perturbative theory [32] or $Q_{\mathrm{tr}} \lesssim 2.5 \Lambda$ for unitarity of the S-matrix [33]. In general the specific regime of $Q_{\mathrm{tr}}$ where the theory breaks down depends on the form of the operator (as well as its UV completion). Since it is not straightforward to derive UV completions for the operators here, we consider $Q_{\mathrm{tr}}=4 \Lambda$ and $Q_{\mathrm{tr}}=4 \pi \Lambda$ as representative of where the EFT assumption begins to suffer from large errors.

We implement three different criteria: $Q_{\operatorname{tr}}<\infty$ (corresponding to the naïve limit), $Q_{\mathrm{tr}}<4 \pi \times \Lambda$, and $Q_{\mathrm{tr}}<4 \Lambda$. More specifically, for a given $\Lambda$, we discard any events in violation and thus rescale the calculated cross section by the fraction of events satisfying this criterion at parton-level. The conditions above on the generated events should not be taken literally; they are only to indicate the size of the error in assuming a single effective operator can describe the relevant physics. This procedure gives conservative constraints, in the sense that any new physics giving rise to the operator is expected to be relevant at these scales. In general, this could lead to even stronger constraints on the model, for example from an enhanced signal in the original channel or from other new signal channels [32-34].

Our results for the operators are shown in figure 3, where the solid lines give the lower limit on $\Lambda$ without any condition on the momentum transfer. When a condition on $Q_{\operatorname{tr}}$ is imposed, this weakens and shifts the bound on $\Lambda$; in addition, low values of $\Lambda$ are no longer excluded, which we interpret as the breakdown of the EFT. This is also illustrated by figure 2, where we show the mono-Higgs cross section when each one of the unitarity conditions above is imposed. For very small $\Lambda$, no events satisfy the condition on $Q_{\mathrm{tr}}$. As $\Lambda$ is increased, more events meet the criterion until the suppression of the cross section with large $\Lambda$ takes over. The excluded region is the range of $\Lambda$ where the cross section is above that observable at the LHC (indicated by the dashed line).

For the weakest condition $Q_{\operatorname{tr}}<4 \pi \Lambda$ a constraint is possible for all operators below DM masses around a few hundred $\mathrm{GeV}$. In the most restrictive case $Q_{\operatorname{tr}}<4 \Lambda$, we find that no bound is possible for the operators in eqs. (3.3), (3.6). For the fermion DM operator in eq. (3.7), a limit for a narrow range in $\Lambda$ is still possible with the strongest $Q_{\mathrm{tr}}$ condition and $8 \mathrm{TeV}$ data, but again no bound is expected at larger masses or with a $14 \mathrm{TeV}$ run. Compared to the results for the $7 / 8 \mathrm{TeV}$ runs of the $\mathrm{LHC}$, the $14 \mathrm{TeV}$ run does not necessarily promise a significant improvement with respect to the issue of unitarity due to the need for a stronger $\mathbb{E}_{T}$ cut to suppress backgrounds.

\section{Dark matter via a $Z^{\prime}$ and heavy Higgs}

Motivated by the mono-Higgs processes discussed in the previous section, we construct a simple model with renormalizable interactions where the relevant states may be produced on-shell. The high-dimension operators considered previously are challenging to UV-complete; however, it is more straightforward to generalize the mono-Higgs process, as shown in figure 1. If the intermediate $Z$ is instead a new $Z^{\prime}$ gauge boson, resonant production is possible; the $Z^{\prime}$ then decays to a Higgs plus an intermediate state which 
decays to a DM pair. Since a SM state decaying to DM is highly constrained, we consider a two-Higgs doublet extension to the standard model with $Z^{\prime} \rightarrow h A^{0}$, where $A^{0}$ is a heavy pseudoscalar with a large branching ratio to dark matter. Below we discuss in more detail the $Z^{\prime}$ coupled to a two-Higgs doublet model (2HDM), which is sufficient to determine the mono-Higgs signal. More model-dependent details of the DM coupling to the pseudoscalar are discussed in section 4.3.

The gauge symmetry of the $\mathrm{SM}$ is extended by a $\mathrm{U}(1)_{Z^{\prime}}$, with a new massive $Z^{\prime}$ gauge boson (see, for example, $[35,36]$ ). We assume that this sector also contains a SM singlet scalar $\phi$ that leads to spontaneous breaking of the symmetry and a $Z^{\prime}$ mass at a scale above electroweak symmetry-breaking. There are many choices for how the SM fermions are charged under the $\mathrm{U}(1)_{Z^{\prime}}$; for simplicity, we assume generation-independent charges for the fermions and that only the right-handed quarks $u_{R}$ are charged. ${ }^{2}$ This allows LHC production of the $Z^{\prime}$, but since the leptons are neutral, avoids potentially stringent constraints from searches for dilepton resonances.

For the Higgs sector we assume a Type 2 two-Higgs-doublet model, where $\Phi_{u}$ couples to up-type quarks and $\Phi_{d}$ couples to down-type quarks and leptons:

$$
-\mathcal{L} \supset y_{u} Q \tilde{\Phi}_{u} \bar{u}+y_{d} Q \Phi_{d} \bar{d}+y_{e} L \Phi_{d} \bar{e}+\text { h.c. }
$$

with hypercharge $Y=1 / 2$ Higgs doublets $\Phi_{u}, \Phi_{d}$ that could have $Z^{\prime}$ charges $z_{u}, z_{d}$. In the case we consider, only $u_{R}$ and $\Phi_{u}$ are charged under $\mathrm{U}(1)_{Z^{\prime}}$. Our convention for the charges are shown in table 2 .

After electroweak symmetry breaking, the Higgs doublets attain vevs $v_{u}$ and $v_{d}$, and in unitary gauge the doublets are parametrized as

$$
\begin{aligned}
\Phi_{d} & =\frac{1}{\sqrt{2}}\left(\begin{array}{c}
-\sin \beta H^{+} \\
v_{d}-\sin \alpha h+\cos \alpha H-i \sin \beta A^{0}
\end{array}\right), \\
\Phi_{u} & =\frac{1}{\sqrt{2}}\left(\begin{array}{c}
\cos \beta H^{+} \\
v_{u}+\cos \alpha h+\sin \alpha H+i \cos \beta A^{0}
\end{array}\right)
\end{aligned}
$$

where $h, H$ are neutral CP-even scalars and $A^{0}$ is a neutral CP-odd scalar. Furthermore, $\tan \beta \equiv v_{u} / v_{d}$, and $\alpha$ is the mixing angle that diagonalizes the $h-H$ mass squared matrix.

We make some simplifying assumptions for the Higgs sector, taking $h$ as the scalar corresponding to the observed Higgs boson with $m_{h} \sim 125 \mathrm{GeV}$. The remaining scalars $H, A^{0}, H^{ \pm}$are assumed to have masses around or above $300 \mathrm{GeV}$, in accordance with $b \rightarrow s \gamma$ constraints [37]. Fits to the observed Higgs couplings from the LHC [38] indicate that a Type $22 \mathrm{HDM}$ is tightly constrained around the alignment limit where $\sin (\beta-\alpha) \rightarrow 1$ (specifically $\beta \rightarrow \alpha+\pi / 2, \alpha \in(-\pi / 2,0)$ ). In this limit, $h$ has SM-like couplings to fermions and gauge bosons. In addition, perturbativity of the top yukawa coupling implies $\tan \beta \gtrsim 0.3$. Hence, we choose to work in the $\alpha-\beta$ parameter space where $\tan \beta \geq 0.3$ and $\alpha=\beta-\pi / 2$.

\footnotetext{
${ }^{2}$ Anomaly cancellation can be achieved with a pair of colored triplet fields which are singlets with respect to $\mathrm{SU}(2)_{L}: \psi_{L}\left(Q_{z}=0, Y=-2 / 3\right)$ and $\psi_{R}\left(Q_{z}=-z_{u}, Y=-2 / 3\right)$ where $z_{u}$ is the $Z^{\prime}$ charge of $u_{R}$.
} 


\begin{tabular}{|c|c|c|c|c|c|}
\hline & $\Phi_{d}$ & $\Phi_{u}$ & $Q_{L}$ & $d_{R}$ & $u_{R}$ \\
\hline $\mathrm{U}(1)_{Z^{\prime}}$ & 0 & $1 / 2$ & 0 & 0 & $1 / 2$ \\
\hline
\end{tabular}

Table 2. SM fermion and scalar $\mathrm{U}(1)_{Z^{\prime}}$ gauge charges. All other SM particles are neutral.

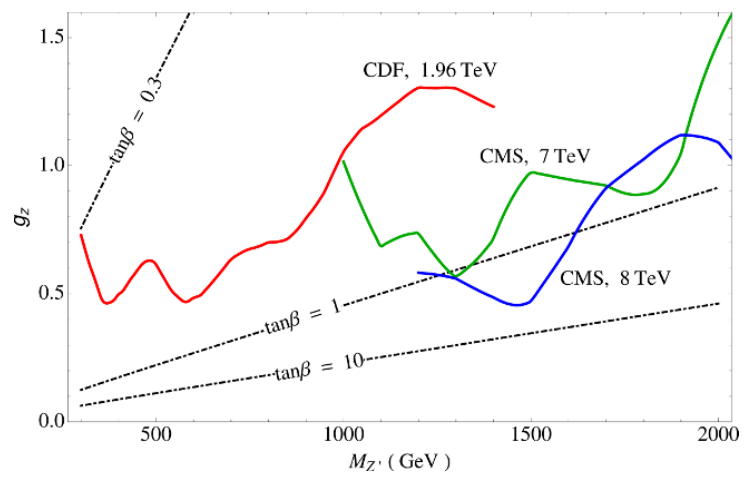

Figure 4. $95 \% \mathrm{CL}$ bounds on the $Z^{\prime}$ coupling $g_{z}$ as a function of $M_{Z^{\prime}}$. The dashed lines are upper bounds from $\rho_{0}$ parameter constraints on $Z-Z^{\prime}$ mixing, given in eq. (4.7), for three values of $\tan \beta=0.3,1,10$. We also show upper limits from dijet resonance searches at the Tevatron and at the LHC; see text for further details.

The Higgs vevs lead to $Z-Z^{\prime}$ mass mixing. Diagonalizing the gauge boson mass matrix, the tree-level masses of the $Z$ and $Z^{\prime}$ bosons are given by

$$
\begin{gathered}
M_{Z}^{2} \approx\left(M_{Z}^{0}\right)^{2}-\epsilon^{2}\left[\left(M_{Z^{\prime}}^{0}\right)^{2}-\left(M_{Z}^{0}\right)^{2}\right] \\
M_{Z^{\prime}}^{2} \approx\left(M_{Z^{\prime}}^{0}\right)^{2}+\epsilon^{2}\left[\left(M_{Z^{\prime}}^{0}\right)^{2}-\left(M_{Z}^{0}\right)^{2}\right],
\end{gathered}
$$

where $\left(M_{Z}^{0}\right)^{2}=g^{2}\left(v_{d}^{2}+v_{u}^{2}\right) /\left(4 \cos ^{2} \theta_{w}\right)$ and $\left(M_{Z^{\prime}}^{0}\right)^{2}=g_{z}^{2}\left(z_{d}^{2} v_{d}^{2}+z_{u}^{2} v_{u}^{2}+z_{\phi}^{2} v_{\phi}^{2}\right)$ are the masssquared values in the absence of mixing. The result above is accurate to order $\epsilon^{2}$, where $\epsilon$ is a small mixing parameter given by

$$
\begin{aligned}
\epsilon & \equiv \frac{1}{M_{Z^{\prime}}^{2}-M_{Z}^{2}} \frac{g g_{z}}{2 \cos \theta_{w}}\left(z_{d} v_{d}^{2}+z_{u} v_{u}^{2}\right) \\
& =\frac{\left(M_{Z}^{0}\right)^{2}}{M_{Z^{\prime}}^{2}-M_{Z}^{2}} \frac{2 g_{z} \cos \theta_{w}}{g} z_{u} \sin ^{2} \beta
\end{aligned}
$$

Finally, the mass eigenstates corresponding to the observed $Z$ boson and the hypothetical $Z^{\prime}$ boson are

$$
\begin{aligned}
Z^{\mu} & \approx W^{3 \mu} \cos \theta_{w}-B_{Y}^{\mu} \sin \theta_{w}+\epsilon B_{Z}^{\mu} \\
Z^{\prime \mu} & \approx B_{Z}^{\mu}-\epsilon\left(W^{3 \mu} \cos \theta_{w}-B_{Y}^{\mu} \sin \theta_{w}\right) .
\end{aligned}
$$

\section{1 $Z^{\prime}$ constraints}

The $Z-Z^{\prime}$ mixing leads to a modification to the $Z$ mass, as shown in eq. (4.3). This in turn affects the relation between the $W$ and $Z$ masses, which is expressed as a deviation 
of the $\rho_{0}$ parameter away from unity:

$$
\rho_{0}=1+\epsilon^{2}\left(\frac{M_{Z^{\prime}}^{2}-M_{Z}^{2}}{M_{Z}^{2}}\right),
$$

Current precision electroweak global fits constrain $\rho_{0}=1.0004_{-0.0004}^{+0.0003}$ [39]. Taking this result at face value, the approximate $95 \%$ upper limit

$$
\rho_{0} \leq 1.0009
$$

implies an upper limit on $g_{z}$ (at fixed $\tan \beta$ and $M_{Z^{\prime}}$ ), shown in figure 4. Although there are also strong LEP constraints on $Z-Z^{\prime}$ mixing from, e.g., precision measurements of dijets/dileptons through a $Z$ resonance, in our case the limits are weak since the coupling to leptons is $\epsilon$-suppressed.

There are additional $g_{z}$ constraints from searches for dijet resonances from $Z^{\prime}$ decay to $q \bar{q}$, also shown in figure 4 . We apply results from Tevatron and LHC studies, with Tevatron results [40] providing coverage for $300 \mathrm{GeV} \leq M_{Z^{\prime}} \leq 1.4 \mathrm{TeV}$. We also apply $95 \% \mathrm{CL}$ upper limits from CMS using $7 \mathrm{TeV}$ [41] and $8 \mathrm{TeV}$ [42] data, ${ }^{3}$ given in a model-independent form in terms of a cross section times acceptance for a narrow resonance decaying to $q \bar{q}$. An upper bound on $g_{z}$ is derived by comparing our detector-level simulation to the published upper limits, assuming that the $Z^{\prime}$ width is fixed for the most part by its decay to quarks:

$$
\Gamma_{Z^{\prime} \rightarrow q \bar{q}} \approx \frac{g_{z}^{2}}{24 \pi} z_{u}^{2} N_{c} M_{Z^{\prime}}
$$

for each light-quark flavor. This is a valid approximation for the model here, assuming that there isn't a significant width for $Z^{\prime}$ decay to other new fermionic modes.

For masses below $\sim 1.3 \mathrm{TeV}$ (exactly the regime that we find the strongest potential mono-Higgs signal) and in particular for large $\tan \beta$, we find that the $\rho_{0}$ constraint on $g_{z}$ is stronger than dijet limits. However, for $\tan \beta \lesssim 0.6$, the dijet constraints dominate even at low masses. For the remainder of the paper, for any given $M_{Z^{\prime}}$ and $\tan \beta$, we will simply assume the coupling $g_{z}$ is the maximum allowed by $\rho_{0}$ and dijet constraints, as given in figure 4.

\subsection{Mono-Higgs signal}

The mono-Higgs signal associated with DM plus Higgs production proceeds through $Z^{\prime} \rightarrow$ $h A^{0}$; the decay width for this to leading order in $\epsilon$ is

$$
\Gamma_{Z^{\prime} \rightarrow h A^{0}}=\left(g_{z} \cos \alpha \cos \beta\right)^{2} \frac{|p|}{24 \pi} \frac{|p|^{2}}{M_{Z^{\prime}}^{2}} .
$$

The center of mass momentum for the decay products is $|p|=\frac{1}{2 M_{Z^{\prime}}} \lambda^{1 / 2}\left(M_{Z^{\prime}}^{2}, m_{h}^{2}, m_{A^{0}}^{2}\right)$, where $\lambda$ is the Källen triangle function. Since only the $\Phi_{u}$ doublet couples directly to the

\footnotetext{
${ }^{3}$ The ATLAS collaboration has also presented 95\% CL upper limits [43, 44], but for a narrow Gaussian in dijet mass distribution, which is not applicable to this case since there is a significant tail to the distribution at lower dijet masses.
} 

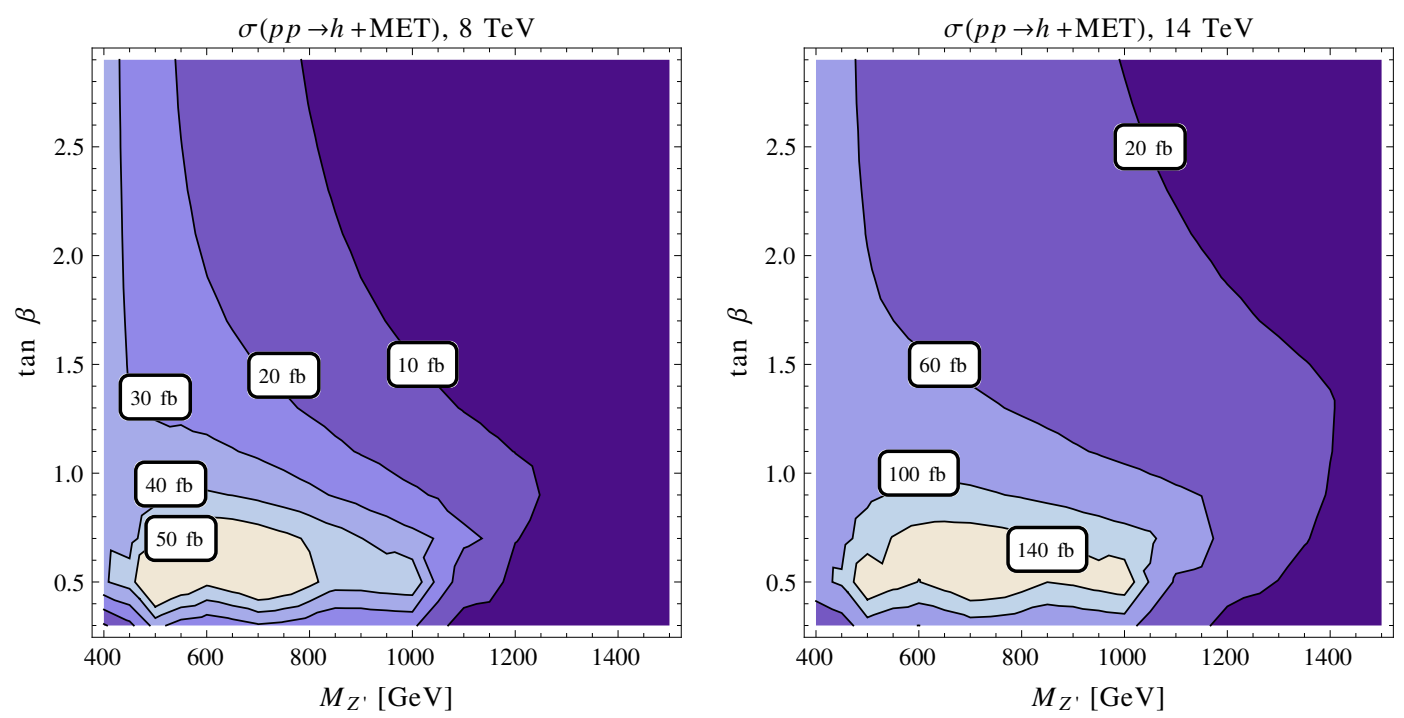

Figure 5. Total cross sections for Higgs+MET, via a new $Z^{\prime}$ gauge boson coupled to a 2HDM, for the LHC at $8 \mathrm{TeV}$ and $14 \mathrm{TeV}$. Both Higgs plus DM production from $Z^{\prime} \rightarrow h A^{0}$ and Higgs plus MET from $Z^{\prime} \rightarrow h Z, Z \rightarrow \nu \bar{\nu}$ are included. The $Z^{\prime}$ gauge coupling is fixed to be its $95 \%$ CL upper limit, as shown in figure 4 .

$Z^{\prime}$, and since the pseudoscalar component of the $\Phi_{u}$ scales with $\cos \beta$, this decay width is suppressed by $1 / \tan ^{2} \beta$ in the limit of large $\tan \beta$. For $\tan \beta<1$, the rate actually increases because the allowed $g_{z}$ from the precision electroweak constraint increases, at least until $\tan \beta \approx 0.6$ when dijet limits take over.

The $Z^{\prime}$ model enjoys an additional source of Higgs plus MET from the decay of $Z^{\prime} \rightarrow$ $h Z$, where the $Z$ decays invisibly. The decay width is

$$
\Gamma_{Z^{\prime} \rightarrow h Z}=\left(g_{z} \cos \alpha \sin \beta\right)^{2} \frac{|p|}{24 \pi}\left(\frac{|p|^{2}}{M_{Z^{\prime}}^{2}}+3 \frac{M_{Z}^{2}}{M_{Z^{\prime}}^{2}}\right)
$$

which grows with smaller $M_{Z^{\prime}}$ due to the $M_{Z}^{2} / M_{Z}^{\prime 2}$ term. At fixed $M_{Z^{\prime}}$, the mono-Higgs rate for this process is almost independent of $\tan \beta$ for $\tan \beta \gtrsim 0.6$. Although the rate naïvely scales as $\sin ^{4} \beta$, this dependence is almost exactly cancelled when we apply the upper limit on $g_{z}$ from $\rho_{0}$, which leads to an upper limit on $g_{z} \propto 1 /\left(\sin ^{2} \beta\right)$. This can also be seen from eqs. (4.4), (4.6). When $\tan \beta \lesssim 0.6$, the constraint on $g_{z}$ is independent of $\tan \beta$ and the width is therefore suppressed by $\sin ^{4} \beta$.

Figure 5 shows the total mono-Higgs cross section at $8 \mathrm{TeV}$ and at $14 \mathrm{TeV}$, as a function of $M_{Z^{\prime}}$ and $\tan \beta$. We have fixed the coupling $g_{z}$ according to its $95 \%$ CL upper bound, as discussed in the previous section. The heavy scalar masses are assumed to be $300 \mathrm{GeV}$ and we take the alignment limit, $\sin (\beta-\alpha)=1$. The branching ratio of $A^{0}$ to dark matter is taken to be $100 \%$. Despite the larger coupling allowed at larger $M_{Z^{\prime}}$, the total cross section eventually falls with $M_{Z^{\prime}}$ due to pdf suppression. For large or small $\tan \beta$, the cross section also falls due to the $(\sin \beta \cos \beta)^{2}$ dependence in the $h A^{0}$ channel. The ratio of the two mono-Higgs rates is shown in figure 6 . Over much of the parameter space we consider, 


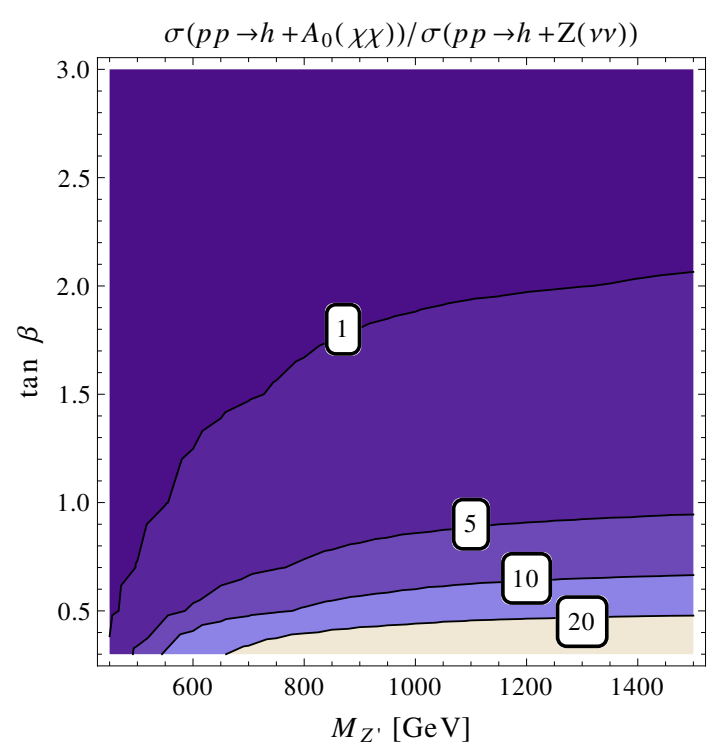

Figure 6. Ratio of the cross sections (or, ratio of the branching ratios) for mono-Higgs from $Z^{\prime} \rightarrow h A^{0}, A^{0} \rightarrow \bar{X} X$ to mono-Higgs from $Z^{\prime} \rightarrow h Z, Z \rightarrow \bar{\nu} \nu$.

the mono-Higgs from $Z^{\prime} \rightarrow h A^{0}$ dominates, however $Z^{\prime} \rightarrow h Z$ is a non-negligible fraction of the total signal and becomes important at low $M_{Z^{\prime}}$ and also at large $\tan \beta$.

We present results for the mono-Higgs reach at the LHC in figure 7. For Run 1 of the LHC (combined $7 \mathrm{TeV}$ and $8 \mathrm{TeV}$ ), we show the three 95\% CL exclusion regions for the $b \bar{b}$ channel with $\mathbb{E}_{T}>120,160$, and $200 \mathrm{GeV}$, where the constrained region increases with MET cut. ${ }^{4}$ For $14 \mathrm{TeV}$ projections, we again find better overall sensitivity with a harder MET cut (taken here to be $\mathbb{E}_{T}>250 \mathrm{GeV}$ ) to reduce SM backgrounds.

The diphoton channel is sensitive to lower cross sections compared to $b \bar{b}$ for a $14 \mathrm{TeV}$ LHC, as evidenced by the reach of this channel for large values of $\tan \beta$. Although our plot cuts off at $\tan \beta=5$, the mono-Higgs cross section is approximately constant for large $\tan \beta$ and the sensitivity can extend to much higher $\tan \beta$. However for much larger $\tan \beta$, direct searches for $H, A^{0}$ would start to be constraining [45], depending on the scalar masses. The diphoton channel also performs worse than expected at large $M_{Z^{\prime}}$. This is because in our detector simulation, the energy resolution for photons deteriorates at higher energies such that the $m_{\gamma \gamma}$ peak is much broader, which limits the signal efficiency. This effect could be reduced by loosening the cut on $m_{\gamma \gamma}$, however the extent to which this would be helpful depends on the actual energy resolution in the experiment.

An appropriate question is whether other $14 \mathrm{TeV}$ searches will potentially also have sensitivity for this model. For example, although data from the next LHC run will improve dijet resonance constraints, this will be mainly at large $M_{Z^{\prime}}$; below $1.5 \mathrm{TeV}$ it will be even more difficult to probe due to the large QCD backgrounds. Here the strongest constraint for our model was the precision electroweak fit for $\rho_{0}$. A somewhat indirect but possibly

\footnotetext{
${ }^{4}$ If we were to use the results of ref. [19], the $8 \mathrm{TeV}$ data would be unconstraining at 95\% CL for almost the entire parameter space. This is partly due to the rather conservatives estimates and also because the cuts are not optimal for our model.
} 


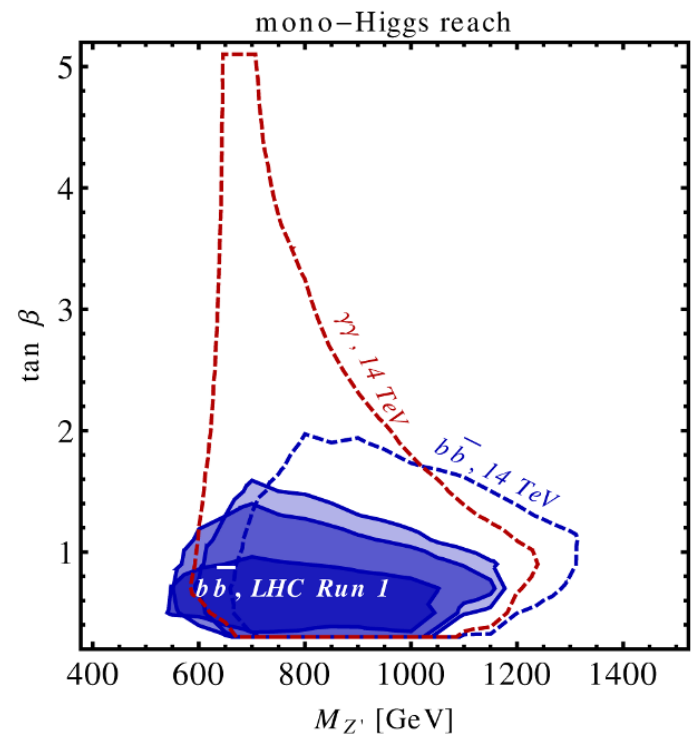

Figure 7. 95\% CL exclusion regions for the parameter space with data from Run 1 of the LHC (7 and $8 \mathrm{TeV}$, total $25 / \mathrm{fb}$ ) for the $b \bar{b}$ channel with MET cuts of 120,160 and $200 \mathrm{GeV}$. Dashed lines give projections for a $14 \mathrm{TeV}$ LHC with $300 / \mathrm{fb}$ integrated luminosity for $b \bar{b}$ and diphoton channels. We only show the parameter space up to $\tan \beta=5$ but the reach for the diphoton channel could extend to somewhat larger $\tan \beta$, since the cross section is approximately constant with $\tan \beta$.

important channel is a direct search for $H, A^{0}$ decay; for example, for $H$ decay to SM fermions, the $14 \mathrm{TeV}$ data could improve the upper limits on $\tan \beta$ significantly for the range of masses relevant here [46].

\subsection{Dark matter coupling to Higgs sector}

To incorporate DM interactions, we have assumed that the CP-odd pseudoscalar $A^{0}$ of the theory possesses a large coupling to DM particles, such that the branching ratio is order one. Here we sketch out some simple models that could give rise to this kind of coupling, reserving more detailed studies for future work.

One possibility is fermion DM; for example, a pseudoscalar interaction can arise in singlet-doublet DM from a coupling to the down-type Higgs. In this model, a singlet $S$ and electroweak doublets $D_{1,2}$ (all singlets under $\mathrm{U}(1)_{Z^{\prime}}$ ) are introduced, with a Lagrangian

$$
-\mathcal{L} \supset \frac{1}{2} M_{S}^{2} S^{2}+M_{D} D_{1} D_{2}+y_{1} S D_{1} \Phi_{d}+y_{2} S \Phi_{d}^{\dagger} D_{2}+\text { h.c. }
$$

The DM is the Majorana fermion that is the lightest mass eigenstate, and we require that it has a mass of at least $m_{h} / 2$ in order to avoid bounds on the invisible width of the Higgs. In general, this state is a mixture of the singlet and the neutral components of the doublets. For more details, see for example refs. [47, 48].

Elastic scattering off quarks can proceed via the exchange of $h$ or $H$, and direct detection constraints severely restrict the parameter space for this model. However, in parts of the parameter space near the "blind spot" where the coupling through the Higgs is suppressed, the direct detection cross sections are small. This cancellation requires $\tan \theta<0$, 
where $y_{1}=y \cos \theta, y_{2}=y \sin \theta$. We find it is possible to obtain large branching ratios of $A^{0}$ to DM while satisfying LUX constraints [49] for parameter values of $y=1.5, \tan \theta=-2$ and masses of $M_{S} \approx 100-200 \mathrm{GeV}$ and $M_{D} \approx 120-180 \mathrm{GeV}$.

For scalar DM, we consider a complex scalar field $X$, written as $X=\frac{1}{\sqrt{2}}\left(X_{1}+i X_{2}\right)$, which is a $\mathrm{SM}$ singlet and has $\mathrm{U}(1)_{Z^{\prime}}$ charge $-1 / 4$. Then the renormalizable interactions of the DM with the Higgs sector are

$$
\begin{aligned}
\mathcal{L} \supset\left(\lambda_{\mathrm{dd}}\left|\Phi_{d}\right|^{2}+\right. & \left.\lambda_{\mathrm{uu}}\left|\Phi_{u}\right|^{2}\right)|X|^{2} \\
& +\left(\lambda_{\mathrm{du}} \Phi_{d}^{\dagger} \Phi_{u} X^{2}+\text { h.c. }\right),
\end{aligned}
$$

with all couplings taken to be real. The mass eigenstates are the real fields fields $X_{1,2}$ with masses $m_{1,2}^{2}=m_{X}^{2} \mp 2 \lambda_{\mathrm{du}} \sin (2 \beta) m_{W}^{2} / g^{2}$, where the overall mass scale $m_{X}^{2}$ is a free parameter. Again, the lightest component is a DM candidate.

The $A^{0}$ can decay through the term $\lambda_{\mathrm{du}} v A^{0} X_{1} X_{2}$. However, this decay is not truly invisible, since the $X_{2}$ can decay to $X_{1} q \bar{q}$ through an off-shell $A$ or $Z^{\prime}$, as well as to $X_{1} \ell^{+} \ell^{-}$ with a somewhat smaller rate. This $X_{2}$ decay will wash out some of the missing energy; however, if the splitting between $X_{2}$ and $X_{1}$ is not too large, these additional jets or leptons are relatively soft. There is some tension for this parameter space, since larger $\lambda_{\mathrm{du}}$ is needed for an $\mathcal{O}(1)$ branching fraction, but at the same time this leads to a larger mass splitting.

Finally, DM scattering off of quarks is through $h$ or $H$ exchange, since $Z^{\prime}$ interactions are inelastic with a large mass splitting. It is possible to satisfy the direct detection limits from LUX if there are cancellations among the couplings $\lambda_{\mathrm{dd}}, \lambda_{\mathrm{uu}}$, and $\lambda_{\mathrm{du}}$ at the $10 \%$ level [50]. We find that couplings of order $|\lambda| \sim 0.1$ and a mass scale of $m_{X} \sim 100 \mathrm{GeV}$ can give rise to the desired features of the model.

\section{Summary and conclusions}

The discovery of a new particle brings with it the prospect of a new signal channel for probing dark matter particle physics. In the search for dark matter, there are already many different potential avenues to its discovery, though so far without conclusive results. The simple question motivating this work is to search for possible models where dark matter production with a Higgs is the dominant discovery mode in the current generation of hadron colliders. For these models we adopted ATLAS results from the combined 7 and $8 \mathrm{TeV}(25 / \mathrm{fb})$ analysis in the $h \rightarrow \bar{b} b$ channel in order to derive constraints, and studied the sensitivity of a $14 \mathrm{TeV}$ LHC in the $\bar{b} b$ as well as diphoton channels.

One way for mono-Higgs to occur is through higher dimension operators coupling dark matter to Higgs doublets and electroweak gauge bosons. LHC constraints applied to the dimension- 7 or -8 operators studied here lead to the naïve conclusion that the cutoff scale $\Lambda$ must be greater than $100-200 \mathrm{GeV}$. However, this is problematic from an effective field theory point of view, since such scales are low compared to the typical momentum transfer in the collider process. We have attempted to quantify the extent to which imposing a unitary constraint gives rise to a reliable (although conservative) bound on the operator. This is possible only for low dark matter masses. 
We also presented a viable simplified model, where the resonant production of a $Z^{\prime}$ decaying to $h A^{0}$ and $h Z$ allows for a potentially observable rate of mono-Higgs. This is primarily possible at low $\tan \beta$, in part because the $Z^{\prime} \rightarrow h A^{0}$ branching fraction is $1 / \tan \beta^{2}$ suppressed. In addition, we require the $Z^{\prime}$ gauge coupling to be near the maximum allowed from precision electroweak fits. Nevertheless, we show there is an interesting part of parameter space for low $\tan \beta$ and $M_{Z^{\prime}}$ around $1 \mathrm{TeV}$, assuming the pseudoscalar $A^{0}$ decays to dark matter $100 \%$ of the time. We briefly discussed possible models that could give rise to this large pseudoscalar to invisible branching ratio. It would be interesting to pursue more detailed model-building work in this direction, taking into account direct detection or relic density considerations.

Acknowledgements: We would like to thank Dan Hooper, Austin Joyce, Björn Penning, Sean Tulin, and Daniel Whiteson for valuable discussion. L.T.W. is supported by a DOE Early Career Award under grant DE-SC0003930. This work was supported in part by the Kavli Institute for Cosmological Physics at the University of Chicago through grant NSF PHY-1125897 and an endowment from the Kavli Foundation and its founder Fred Kavli.

Open Access. This article is distributed under the terms of the Creative Commons Attribution License (CC-BY 4.0), which permits any use, distribution and reproduction in any medium, provided the original author(s) and source are credited.

\section{References}

[1] G. Jungman, M. Kamionkowski and K. Griest, Supersymmetric dark matter, Phys. Rept. 267 (1996) 195 [hep-ph/9506380] [INSPIRE].

[2] M. Beltrán, D. Hooper, E.W. Kolb, Z.A.C. Krusberg and T.M.P. Tait, Maverick dark matter at colliders, JHEP 09 (2010) 037 [arXiv: 1002.4137] [INSPIRE].

[3] P.J. Fox, R. Harnik, J. Kopp and Y. Tsai, LEP shines light on dark matter, Phys. Rev. D 84 (2011) 014028 [arXiv: 1103.0240] [INSPIRE].

[4] P.J. Fox, R. Harnik, J. Kopp and Y. Tsai, Missing energy signatures of dark matter at the LHC, Phys. Rev. D 85 (2012) 056011 [arXiv:1109.4398] [InSPIRE].

[5] A. Rajaraman, W. Shepherd, T.M.P. Tait and A.M. Wijangco, LHC bounds on interactions of dark matter, Phys. Rev. D 84 (2011) 095013 [arXiv: 1108.1196] [INSPIRE].

[6] ATLAS collaboration, Search for dark matter candidates and large extra dimensions in events with a jet and missing transverse momentum with the ATLAS detector, JHEP 04 (2013) 075 [arXiv:1210.4491] [INSPIRE].

[7] CMS collaboration, Search for dark matter and large extra dimensions in monojet events in pp collisions at $\sqrt{s}=7 \mathrm{TeV}$, JHEP 09 (2012) 094 [arXiv:1206.5663] [INSPIRE].

[8] L.M. Carpenter, A. Nelson, C. Shimmin, T.M.P. Tait and D. Whiteson, Collider searches for dark matter in events with a $Z$ boson and missing energy, Phys. Rev. D 87 (2013) 074005 [arXiv: 1212.3352] [INSPIRE].

[9] ATLAS collaboration, Search for dark matter in events with a hadronically decaying $W$ or $Z$ boson and missing transverse momentum in pp collisions at $\sqrt{s}=8 \mathrm{TeV}$ with the ATLAS detector, Phys. Rev. Lett. 112 (2014) 041802 [arXiv: 1309.4017] [INSPIRE]. 
[10] T. Lin, E.W. Kolb and L.-T. Wang, Probing dark matter couplings to top and bottom quarks at the LHC, Phys. Rev. D 88 (2013) 063510 [arXiv:1303.6638] [InSPIRE].

[11] H. An, X. Ji and L.-T. Wang, Light dark matter and $Z^{\prime}$ dark force at colliders, JHEP 07 (2012) 182 [arXiv:1202.2894] [INSPIRE].

[12] Y. Bai and J. Berger, Fermion portal dark matter, JHEP 11 (2013) 171 [arXiv:1308.0612] [INSPIRE].

[13] S. Chang, R. Edezhath, J. Hutchinson and M. Luty, Effective WIMPs, Phys. Rev. D 89 (2014) 015011 [arXiv: 1307.8120] [INSPIRE].

[14] A. DiFranzo, K.I. Nagao, A. Rajaraman and T.M.P. Tait, Simplified models for dark matter interacting with quarks, JHEP 11 (2013) 014 [arXiv:1308.2679] [INSPIRE].

[15] M. Papucci, A. Vichi and K.M. Zurek, Monojet versus rest of the world I: t-channel models, arXiv: 1402.2285 [INSPIRE].

[16] ATLAS collaboration, Observation of a new particle in the search for the standard model Higgs boson with the ATLAS detector at the LHC, Phys. Lett. B 716 (2012) 1 [arXiv:1207.7214] [INSPIRE].

[17] CMS collaboration, Observation of a new boson at a mass of $125 \mathrm{GeV}$ with the CMS experiment at the LHC, Phys. Lett. B $\mathbf{7 1 6}$ (2012) 30 [arXiv:1207.7235] [INSPIRE].

[18] A.A. Petrov and W. Shepherd, Searching for dark matter at LHC with Mono-Higgs production, Phys. Lett. B 730 (2014) 178 [arXiv:1311.1511] [INSPIRE].

[19] L. Carpenter et al., Mono-Higgs: a new collider probe of dark matter, Phys. Rev. D 89 (2014) 075017 [arXiv: 1312.2592] [INSPIRE].

[20] C.P. Burgess, M. Pospelov and T. ter Veldhuis, The minimal model of nonbaryonic dark matter: a singlet scalar, Nucl. Phys. B 619 (2001) 709 [hep-ph/0011335] [INSPIRE].

[21] A. Djouadi, O. Lebedev, Y. Mambrini and J. Quevillon, Implications of LHC searches for Higgs-portal dark matter, Phys. Lett. B 709 (2012) 65 [arXiv:1112.3299] [INSPIRE].

[22] A. Greljo, J. Julio, J.F. Kamenik, C. Smith and J. Zupan, Constraining Higgs mediated dark matter interactions, JHEP 11 (2013) 190 [arXiv: 1309.3561] [INSPIRE].

[23] LhC Higgs Cross Section Working Group collaboration, S. Heinemeyer et al., Handbook of LHC Higgs cross sections: 3. Higgs properties, arXiv:1307.1347 [INSPIRE].

[24] ATLAS collaboration, Search for the bb decay of the standard model Higgs boson in associated W/ZH production with the ATLAS detector, ATLAS-CONF-2013-079 (2013).

[25] A. Alloul, N.D. Christensen, C. Degrande, C. Duhr and B. Fuks, FeynRules 2.0 - A complete toolbox for tree-level phenomenology, arXiv:1310.1921 [INSPIRE].

[26] J. Alwall, M. Herquet, F. Maltoni, O. Mattelaer and T. Stelzer, MadGraph 5: going beyond, JHEP 06 (2011) 128 [arXiv:1106.0522] [INSPIRE].

[27] T. Sjöstrand, S. Mrenna and P.Z. Skands, PYTHIA 6.4 physics and manual, JHEP 05 (2006) 026 [hep-ph/0603175] [INSPIRE].

[28] DELPHES 3 collaboration, J. de Favereau et al., DELPHES 3, a modular framework for fast simulation of a generic collider experiment, JHEP 02 (2014) 057 [arXiv: 1307.6346] [INSPIRE]. 
[29] CMS collaboration, Search for the standard model Higgs boson produced in association with a W or a $Z$ boson and decaying to bottom quarks, Phys. Rev. D 89 (2014) 012003 [arXiv:1310.3687] [INSPIRE].

[30] J.-Y. Chen, E.W. Kolb and L.-T. Wang, Dark matter coupling to electroweak gauge and Higgs bosons: an effective field theory approach, Phys. Dark Univ. 2 (2013) 200 [arXiv: 1305.0021] [INSPIRE].

[31] M.A. Fedderke, E.W. Kolb, T. Lin and L.-T. Wang, $\gamma$-ray constraints on dark-matter annihilation to electroweak gauge and Higgs bosons, arXiv: 1310.6047 [INSPIRE].

[32] G. Busoni, A. De Simone, E. Morgante and A. Riotto, On the validity of the effective field theory for dark matter searches at the LHC, Phys. Lett. B 728 (2014) 412 [arXiv: 1307.2253] [INSPIRE].

[33] I.M. Shoemaker and L. Vecchi, Unitarity and monojet bounds on models for DAMA, CoGeNT and CRESST-II, Phys. Rev. D 86 (2012) 015023 [arXiv:1112.5457] [INSPIRE].

[34] O. Buchmueller, M.J. Dolan and C. McCabe, Beyond effective field theory for dark matter searches at the LHC, JHEP 01 (2014) 025 [arXiv: 1308.6799] [INSPIRE].

[35] P. Langacker, The physics of heavy $Z^{\prime}$ gauge bosons, Rev. Mod. Phys. 81 (2009) 1199 [arXiv:0801.1345] [INSPIRE].

[36] M.S. Carena, A. Daleo, B.A. Dobrescu and T.M.P. Tait, $Z^{\prime}$ gauge bosons at the Tevatron, Phys. Rev. D 70 (2004) 093009 [hep-ph/0408098] [InSPIRE].

[37] G.C. Branco et al., Theory and phenomenology of two-Higgs-doublet models, Phys. Rept. 516 (2012) 1 [arXiv: 1106.0034] [INSPIRE].

[38] N. Craig, J. Galloway and S. Thomas, Searching for signs of the second Higgs doublet, arXiv:1305.2424 [INSPIRE].

[39] Particle Data Group collaboration, J. Beringer et al., Review of particle physics, Phys. Rev. D 86 (2012) 010001 [INSPIRE].

[40] CDF collaboration, T. Aaltonen et al., Search for new particles decaying into dijets in proton-antiproton collisions at $\sqrt{s}=1.96 \mathrm{TeV}$, Phys. Rev. D 79 (2009) 112002 [arXiv: 0812.4036] [INSPIRE].

[41] CMS collaboration, Search for narrow resonances and quantum black holes in inclusive and b-tagged dijet mass spectra from pp collisions at $\sqrt{s}=7$ TeV, JHEP 01 (2013) 013 [arXiv: 1210.2387] [INSPIRE].

[42] CMS collaboration, Search for narrow resonances using the dijet mass spectrum with $19.6 \mathrm{fb}^{-1}$ of pp collisions at $\sqrt{\mathrm{s}}=8 \mathrm{TeV}$, CMS-PAS-EXO-12-059 (2012).

[43] ATLAS collaboration, Search for new physics in the dijet mass distribution using $1 \mathrm{fb}^{-1}$ of pp collision data at $\sqrt{s}=7 \mathrm{TeV}$ collected by the ATLAS detector, Phys. Lett. B 708 (2012) 37 [arXiv: 1108.6311] [INSPIRE].

[44] ATLAS collaboration, Search for new phenomena in the dijet mass distribution updated using $13.0 \mathrm{fb}^{-1}$ of pp collisions at $\sqrt{s}=8 \mathrm{TeV}$ collected by the ATLAS Detector, ATLAS-CONF-2012-148 (2012).

[45] CMS collaboration, Higgs to tau tau (MSSM), CMS-PAS-HIG-13-021 (2013).

[46] A. Arbey, M. Battaglia and F. Mahmoudi, Supersymmetric heavy Higgs bosons at the LHC, Phys. Rev. D 88 (2013) 015007 [arXiv: 1303.7450] [INSPIRE]. 
[47] T. Cohen, J. Kearney, A. Pierce and D. Tucker-Smith, Singlet-doublet dark matter, Phys. Rev. D 85 (2012) 075003 [arXiv: 1109.2604] [INSPIRE].

[48] C. Cheung and D. Sanford, Simplified models of mixed dark matter, JCAP 02 (2014) 011 [arXiv:1311.5896] [INSPIRE].

[49] LUX collaboration, D.S. Akerib et al., First results from the LUX dark matter experiment at the Sanford Underground Research Facility, Phys. Rev. Lett. 112 (2014) 091303 [arXiv: 1310.8214] [INSPIRE].

[50] X.-G. He and J. Tandean, Low-mass dark-matter hint from CDMS II, Higgs boson at the LHC and darkon models, Phys. Rev. D 88 (2013) 013020 [arXiv: 1304.6058] [INSPIRE]. 